\title{
Thermal properties of time machines
}

\author{
Pedro F. González-Díaz \\ Colina de los Chopos, Instituto de Física Fundamental,Consejo Superior de Investigaciones Científicas, \\ Serrano 121, 28006 Madrid, Spain
}

(Received 19 March 2012; published 24 May 2012)

\begin{abstract}
Connections between universes through tunneling space-times could make the multiverse a physical entity able to be observed from our own single universe. In this paper we first study the thermal properties of the static Klein-bottle holes and then consider one of the potentially observable effects from wormholes, ringholes, and nonorientable tunnelings when they are converted into time machines connecting other universes with ours own, that is a randomly varying in space and time thermal radiation which, with an unpredictable cadence, randomly manifested to a far observer as a short, occasional pulse with very high intensity and fluence which would be made of black body phantom or ordinary radiation. We discuss the odds for these bursts of thermal radiation to be eventually observable.
\end{abstract}

DOI: 10.1103/PhysRevD.85.105026

PACS numbers: 03.70.+k, 98.80.Jk

\section{INTRODUCTION}

Pathological space-time tunneling solutions to Einstein equations which can be converted into time machines have been studied during the last 23 years or so. Among them one should mention the seminal Morris-Thorne-Yurtsever Lorentzian wormholes [1], the ringholes [2] and the Kleinbottle holes [3]. These tunneling solutions were first considered to be quantum-mechanically unstable as far as all of them were relatable to the prototypical Misner space where the existence of a chronological horizon drives a catastrophic creation of particles, so inducing the regularized stress-energy tensor to diverge [4]. In fact, one can simply generate these solutions by simply replacing the planes of Misner space for, respectively, spheres and orientable and nonorientable tori [4]. The generalization of this result led Hawking to conjecture his so-called chronology protection [5] according to which quantum mechanics should preclude the existence of all pathological solutions leading to the formation of closed timelike curves. However, it was later shown by Li and Gott [6] that there exists a self-consistent conformal vacuum where all time machines become stable so violating Hawking's conjecture. Also somehow contravening that conjecture was some work [7] showing that all space-time time-machine constructs become quantum-mechanically stable at the Planck scale, so allowing the quantum vacuum spacetime foam to exist.

On the other hand, it is sometimes objected that because our observations are limited to a single universe (e.g., a Hubble volume) then the existence of other universes or of the multiverse as a whole cannot be checked, and so their existence cannot be considered a proper scientific hypothesis. Even taking into account the fact that future observers will see a larger particle horizon, and so have access to a bigger volume of space, most regions of the multiverse (at least in the eternal inflationary model) can never be observed, even in principle. While this may indeed preclude direct confirmation of the multiverse hypothesis, it does not rule out the possibility that it may be tested indirectly. Almost all scientists and philosophers accept the general principle that the prediction of unobservable entities is an acceptable scientific hypothesis if those entities stem from a theory that has otherwise testable consequences. Thus, neither observations of interuniversal connections [8] or interuniverse quantum entangled states [9] nor effects in our universe of distinct boundary conditions induced by the presence of other universes [10] can be disregarded as potential ways to prove or disprove the existence of the multiverse.

It has been recently considered [10] that in order to make the multiverse a rather physical idea, the universe we live in ought to be multiply connected through the rest of universes in the multiverse by means of a network mechanism of connecting tunnelings whose optical properties [8] lead to signatures left in our universe which will characterize every type of space-time tunnel and could be used in order to make the multiverse an observable entity [8-10]. Moreover, full independence of the space-times belonging to different universes require that the two mouths of every connecting tunnel move relative to each other with an arbitrary mutual acceleration, so converting the spacetime tunnelings into true time machines without any predictable specifications on the final destination time and location, so producing completely arbitrary closed timelike curves (CTCs) [8].

Another possible way to make evident the existence of other universes could be based on the thermal properties that all the space-time tunnels possess. It has been hitherto shown that static wormholes [11] and ringholes [12] should be expected to quantum-mechanically emit thermal radiation, and that this radiation could perhaps be eventually observed. In this paper, once we check that Klein-bottle holes also quantum-mechanically emit thermal radiation, we investigate the effects that the mutual acceleration between the two tunnel mouths, which gives rise to the 
formation of the random CTCs, has in the thermal radiation that all the holes emit. It will be seen that the above time machines connecting two individual universes deliver in at least one of such universes an arbitrary thermal radiation characterized by a Doppler-shifted temperature which oscillates from a very low value to a very large one in a random way which is utterly arbitrary and unpredictable, too. The surviving prediction is then that there will be fully random, eventual outbursts of thermal ordinary and/or phantom radiation coming from the furthest regions of our universe which, if the tunnels are big enough, could eventually become unmistakable proofs for the existence of other universes. These outbursts could be ultimately converted into true explosions which would be even quite more energetic than the final explosion of primordial black holes [13], though they might leave an ultimate nonzeroenergy remnant in the case of ringholes and Klein-bottle holes.

We outline this paper as follows. In Sec. II we complete the study of static tunnels by establishing the set of thermal properties of static Klein-bottle holes, including its temperature, entropy and the laws of Klein-bottle hole thermodynamics. The effects that converting the different tunnels into interuniverse time machines have on their thermal properties are evaluated in Sec. III, where it is predicted that such effects would eventually produce random outbursts of thermal ordinary and/or phantom radiation which, if the holes are big enough, could eventually become extremely energetic explosions which could be potentially observable. Finally, in Sec. IV we summarize and add some further comments.

\section{THERMAL RADIATION FROM STATIC KLEIN-BOTTLE HOLES}

Klein-bottle holes could lead to the most suitable tunneling mechanism in order for mutually connecting a couple of parallel or otherwise independent universes, as those holes do not preserve any orientability of a universe with respect to the other. In this section we shall derive expressions for the temperatures and entropy that characterize the static Klein-bottle holes. The static metric for a Klein-bottle hole was derived in Ref. [3] and reads (See Fig. 1)

$$
\begin{aligned}
d s^{2}= & -e^{2 \Phi} d t^{2}+\theta\left(2 \pi-\varphi_{1}\right)\left(\frac{d r_{1}^{2}}{1-K\left(b_{1}\right) / b_{1}}+d \Omega_{1}^{2}\right) \\
& +\theta\left(\varphi_{1}-2 \pi\right)\left(\frac{d r_{2}^{2}}{1-K\left(b_{2}\right) / b_{2}}+d \Omega_{2}^{2}\right),
\end{aligned}
$$

where $\Phi$ and $K\left(b_{i}\right)$ are the shift and lapse functions [1], respectively, the $\theta(x)$ 's are the step Heaviside function [14], with $\theta(x)=1$ for $x>0$ and $\theta(x)=0$ for $x<0$, $d \Omega_{i}^{2}$ 's for $0 \leq \varphi_{1} \leq 2 \pi$ is given by

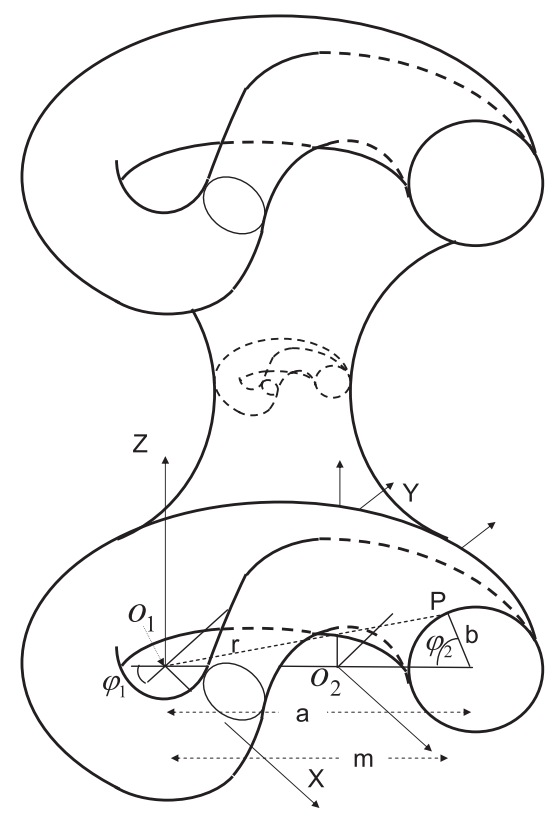

FIG. 1. Pictorial representation of a Klein-bottle hole showing the nonorientable topology of its throat and some of the parameters in terms of which metric (1) is defined.

$$
\begin{aligned}
d \Omega_{1}^{2}= & \left\{m_{1}^{2}+\frac{1}{4}\left[M_{1}\left(a_{1}-C_{1}\right)+N_{1}\left(b_{1}-D_{1}\right)\right]\right\} d \varphi_{1}^{2} \\
& +b_{1}^{2} d \varphi_{2}^{2}-b_{1} \sqrt{\left(a_{1}-C_{1}\right)\left(A_{1}-a_{1}\right)} \sin \varphi_{2} d \varphi_{1} d \varphi_{2}
\end{aligned}
$$

in which

$$
\begin{aligned}
& M_{1}=A_{1}-a_{1}-\left(B_{1}-b_{1}\right) \cos \varphi_{2} \\
& N_{1}=B_{1}-b_{1}-\left(A_{1}-a_{1}\right) \cos \varphi_{2} \\
& m_{i}=a_{i}-b_{i} \cos \varphi_{2}, \quad i=1,2 .
\end{aligned}
$$

Now, for $2 \pi \leq \varphi_{1} \leq 3 \pi$

$$
\begin{aligned}
d \Omega_{2}^{2}= & \left\{m_{2}^{2}+\frac{1}{4}\left[M_{2}\left(a_{2}-A_{2}\right)+N_{2}\left(b_{2}-B_{2}\right)\right]\right\} d \varphi_{2}^{2} \\
& +b_{2}^{2} d \varphi_{2}^{2}-b_{2} \sqrt{\left(a_{2}-A_{2}\right)\left(C_{2}-a_{2}\right)} \sin \varphi_{2} d \varphi_{1} d \varphi_{2},
\end{aligned}
$$

where in this case

$$
\begin{aligned}
& M_{2}=C_{2}-a_{2}-\left(D_{2}-b_{2}\right) \cos \varphi_{2} \\
& N_{2}=D_{2}-b_{2}-\left(C_{2}-a_{2}\right) \cos \varphi_{2} .
\end{aligned}
$$

Finally we have

$$
r_{1}=\sqrt{a_{1}^{2}+b_{1}^{2}-2 a_{1} b_{1} \cos \varphi_{2}}
$$




$$
r_{2}=\sqrt{a_{2}^{2}+b_{2}^{2}+2 a_{2} b_{2} \cos \varphi_{2}},
$$

where we have extended the range of the angular coordinate $\varphi_{1}$ to also encompass values continuously running from $2 \pi$ to $3 \pi$ and $A_{i}, B_{i}, C_{i}$ and $D_{i}, i=1,2$, are given sets of adjustable parameters which are arbitrary unless for the conditions $A_{1}>C_{1}, B_{1}>D_{1}, A_{1}>B_{1}$ and $C_{1}>C_{1}$ for the angular interval $0 \leq \varphi_{1} \leq 2 \pi$, whereas for $2 \pi \leq$ $\varphi_{1} \leq 3 \pi$ we must have $C_{2}>A_{2}, D_{2}>B_{2}, C_{2}>D_{2}$ and $A_{2}>B_{2}$, with $D_{2}=B_{1}, \quad B_{2}=D_{1} \quad$ and $\quad A_{1}-C_{1}=$ $A_{2}+C_{2}$, with $A_{1}-C_{1}>2 A_{2} . a_{i}$ and $b_{i}$ are the radius of the circumference generated by the circular axis of the Klein bottle torus and that of a Klein bottle section, respectively, with $a_{i}>b_{i}$. Metric (1) is defined for $0 \leq t \leq$ $\infty, a_{i}-b_{i} \leq r_{i} \leq a_{i}+b_{i}$ and the angles (see Fig. 1) $0 \leq$ $\varphi_{1}, \varphi_{2} \leq 2 \pi$.

Thus, according to the discussion made when introducing the process of thermal emission in ringholes in Ref. [2], since the $\varphi_{2}$-angular horizons at $\varphi_{2}^{i c}$ and $2 \pi-\varphi_{2}^{i c}$, with $i=1,2$, in Klein-bottle holes are placed exactly at the same positions, along the respective $\varphi_{1}$-intervals, as in the case of ringholes, for Klein-bottle holes one should expect the existence of radiation sources at positive and negative temperatures along the same $\varphi_{2}$-intervals as in the ringhole case; that is, there would be a source at negative temperature radiating phantom energy for the interval $2 \pi-\varphi_{2}^{c 1}>$ $\varphi_{2}>\varphi_{2}^{c 1}=\arccos \left(b_{1} / a_{1}\right)$, and a source at positive temperature for ordinary radiation in the interval $-\varphi_{2}^{c 1}<$ $\varphi_{2}<\varphi_{2}^{c 1}=\arccos \left(b_{1} / a_{1}\right)$, both along the $\varphi_{1}$ interval from 0 to $2 \pi$; in addition there will be as well a source at negative temperature radiating phantom energy for the interval $2 \pi-\varphi_{2}^{c 2}>\varphi_{2}>\varphi_{2}^{c 2}=\arccos \left(b_{2} / a_{2}\right)$, and a source at positive temperature radiating ordinary energy for $-\varphi_{2}^{c 2}<\varphi_{2}<\varphi_{2}^{c 2}=\arccos \left(b_{2} / a_{2}\right)$, both along the $\varphi_{1}$-interval from $2 \pi$ to $3 \pi$ that makes nonorientable the manifold.

An estimate of these temperatures along the two $\varphi_{1}$-intervals of the nonorientable manifold may in turn be obtained by estimating their surface gravities, which can be derived by generalizing the expression for surface gravity in the case of spherical symmetry [11] to that for nonorientable toroidal symmetry. In the present case, this can be simply made by just replacing the radial coordinate $r$ of the spherical case for the distances $\overline{O P}$ (see Fig. 1 and Eqs. (9) and (10)), $r_{i}=\sqrt{a_{i}^{2}+b_{i}^{2}+(-1)^{i} 2 a_{i} b_{i} \cos \varphi_{2}}$, with $i=1,2$. Using then the general procedure described in Ref. [11], we finally have

$$
\begin{aligned}
& \kappa_{1} \propto \frac{b_{01}^{2}}{2 b_{1} r_{1}^{2}}-2 \pi r_{1}\left(\epsilon_{1}-p_{1}\right) \\
& =\frac{b_{01}^{2}}{2 b_{1} r_{1}^{2}}-\frac{c^{4} b_{01}^{2} n_{1}^{2}}{8 G b_{1}^{3} r_{1}} \times\left\{\frac{\frac{8 m_{1}}{a_{1}}-\left(\frac{A_{1}-C_{1}}{a_{1}}\right) \cos ^{2}\left(\frac{\varphi_{1}}{4}\right)+\frac{m_{1}^{(0)}}{m_{1}}+\frac{n_{1}^{(0)}}{n_{1}}-2}{4\left\{m_{1}^{2}+\frac{1}{4}\left[M_{1}\left(A_{1}-C_{1}\right)+N_{1}\left(B_{1}-D_{1}\right) \cos ^{2}\left(\frac{\varphi_{1}}{4}\right)\right\}\right.}+\frac{2\left(1+\sin \varphi_{2}\right)}{n_{1} b_{1}}-\frac{4 \cos \left(\frac{\varphi_{1}}{2}\right) \sin \varphi_{2}}{\left.\left(A_{1}-C_{1}\right) m_{1} \sin ^{2}\left(\frac{\varphi_{1}}{2}\right)\right\}}\right. \\
& \kappa_{2} \propto \frac{b_{02}^{2}}{2 b_{2} r_{2}^{2}}-2 \pi r_{2}\left(\epsilon_{2}-p_{2}\right) \\
& \quad=\frac{b_{02}^{2}}{2 b_{2} r_{2}^{2}}-\frac{c^{4} b_{02}^{2} n_{2}^{2}}{8 G b_{2}^{3} r_{2}} \times\left\{\frac{\frac{8 m_{2}}{a_{2}}-\left(\frac{A_{2}-C_{2}}{a_{2}}\right) \cos ^{2}\left(\frac{\varphi_{1}}{4}\right)+\frac{m_{2}^{(0)}}{m_{2}}+\frac{n_{2}^{(0)}}{n_{2}}-2}{4\left\{m_{2}^{2}+\frac{1}{4}\left[M_{2}\left(A_{2}-C_{2}\right)+N_{2}\left(B_{2}-D_{2}\right) \cos ^{2}\left(\frac{\varphi_{1}}{4}\right)\right\}\right.}+\frac{2\left(1+\sin \varphi_{2}\right)}{n_{2} b_{2}}-\frac{4 \cos \left(\frac{\varphi_{2}}{2}\right) \sin \varphi_{2}}{\left(A_{2}-C_{2}\right) m_{2} \sin 2^{2}\left(\frac{\varphi_{1}}{2}\right)}\right\},
\end{aligned}
$$

with $\epsilon_{i}$ and $p_{i}$ the energy density and the radial pressure, respectively, corresponding to the given $\varphi_{1}$ interval, $i=1$, 2. Now, for the most natural situation in which the absolute value of the radii $a_{i}$ is sufficiently larger than the radii $b_{i}$, since the combination $\epsilon_{i}-p_{i}$ becomes negative in the regions $2 \pi-\varphi_{2}^{c i}>\varphi_{2}>\varphi_{2}^{c i}=\arccos \left(b_{i} / a_{i}\right)$, then the corresponding surface gravities will be definite positive in such regions and, since the radiation temperature is generally given by $T \propto-\left.\kappa\right|_{b=b_{0 i}}$, it follows that that temperature will in fact be negative for the intervals $2 \pi-\varphi_{2}^{c i}>\varphi_{2}>\varphi_{2}^{c i}=\arccos \left(b_{i} / a_{i}\right)$. On the contrary, the combination $\epsilon_{2}-p_{2}$ is positive in the regions $-\varphi_{2}^{c 1}<\varphi_{2}<\varphi_{2}^{c 1}=\arccos \left(b_{1} / a_{1}\right), \quad$ and therefore the associated surface gravities will be definite negative and the temperatures positive.

We have in this way obtained that, similar to the case of ringholes [12], for $\kappa_{i} \simeq-2 \pi r_{i}\left(\epsilon-p_{r}\right)$ : (i) whereas the temperature on the embedding surface that flares outward is always negative, that on the flaring inward embedding surface is always positive and its absolute value is either larger or smaller than that for the negative temperature, depending on the nature of the surrounding vacuum, and (ii) in spite of that, because the involved negativetemperature system and the positive-temperature system can never come in contact in the present case (because the temperature again vanishes at the two angular horizons), 
and hence the former system is not definite hotter or colder than the latter one [15], at least at the first stages of a large- $a_{i}$ Klein-bottle hole evaporation, the intensity and energy of the radiation pulses emitted at the positive temperature may once again overcompensate those generated at a negative temperature or vice versa, so satisfying the generalized quantum interest conjecture as well [12].

It also follows that, as the above overall thermal process at combined positive- and negative-temperature emission progresses, the Klein-bottle hole may be converted into either some sort of nonorientable wormhole in the sense that the surface embedding it would tend to only flare outward (that precisely happening when $a_{i}$ becomes exactly equal to $b_{i}$ [see also Fig. 2(a)]), or some sort of nonorientable black hole in the sense that the embedding surface tended to just flare inward (this taking place when $a_{i}=-b_{i}$ [see also Fig. 2(b)]). These limiting geometric Klein-bottle hole configurations either can only continue emitting phantom radiation at a negative temperature as far as the usual quantum interest conjecture [16] is violated, such as what happens with spherically symmetric wormholes, or can emit ordinary radiation at a positive temperature such as what happens with spherically symmetric black holes.

It is in this sense that also the Klein-bottle holes with these limiting geometries are equivalent either to wormholes or to black holes. There is still another aspect in which wormholes or black holes and the above limiting Klein-bottle hole configurations are again equivalent. It is in that both types of tunneling (wormholes and limiting Klein-bottle holes with $a_{i}=b_{i}$ ) and both types of compact space-time objects (black holes and limiting Klein-bottle holes with $a_{i}=-b_{i}$ ) all show the same optical lensing gravitational signature on the sky when light coming to us from a luminous object is placed behind them, along the line of sight, i.e., a single glowing ring of the kind already considered by Shatskiy for single wormholes [17]. Unfortunately, such bright rings are not at all distinguishable from e.g., Einstein rings generated by, e.g., galaxies, or that is produced by stars with negative energy.

We finally consider entropy expressions for Klein-bottle holes. Thus, according to the known principles of gravitational thermodynamics [18], the one fourth of the surface area of the nonorientable torus making the throat of the Klein-bottle hole is expected to provide us with the Klein-bottle hole entropy expressions, $S_{K H}^{i}$ with $i=1,2$ [18]. Thus, we would have

$$
S_{K H}^{i}=\frac{b_{i}}{4 \ell_{P}^{2}} \int_{0}^{2 \pi} d \varphi_{1} \int_{0}^{2 \pi} d \varphi_{2} m_{i}=\frac{\pi^{2} a_{i} b_{i}}{\ell_{P}^{2}}
$$

which are similar to that for ringholes [12].

We notice that even though neither ringholes nor Kleinbottle holes show any event horizon, they must have a trapping horizon which is nothing but that for spherically symmetric wormholes [11] when generalized to toroidal

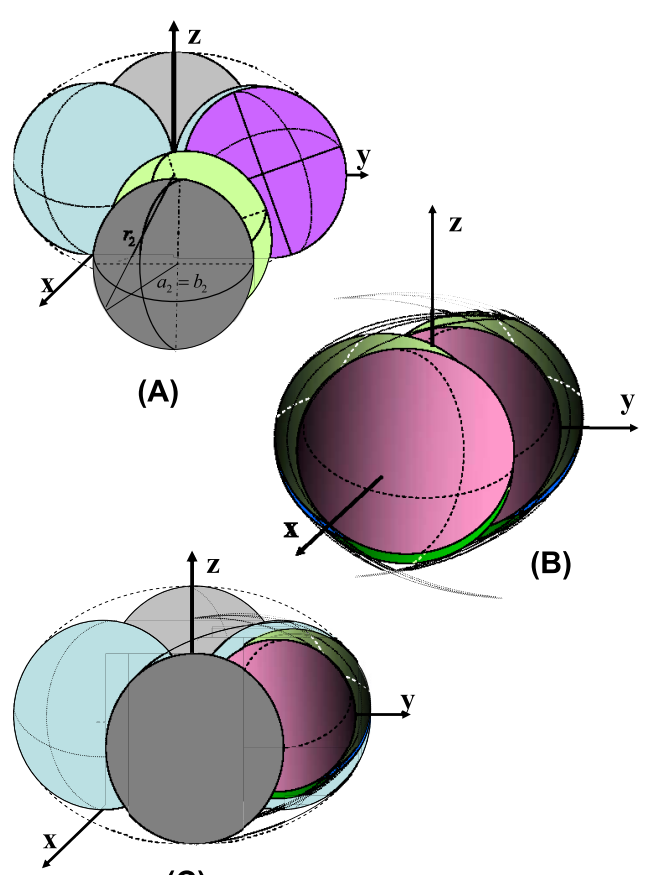

(C)

FIG. 2 (color online). Limiting Klein-bottle holes in which $a_{i}^{2}=b_{i}^{2}, i=1,2$. We have then three possible final situations. (A) if both $a_{1}=b_{1}$ and $a_{2}=b_{2}$, then the process of thermal emission of positive and negative radiation tends to that limiting geometry where all ordinary energy contributing to the Kleinbottle hole with $a_{i}>b_{i}$ is exhausted, leaving a double geometry which is equivalent to that of a couple of wormholes in that the surface embedding them flares outward but not inward, and are both therefore made out of exotic, phantomlike energy characterized by a negative temperature; (B) if both $a_{1}=-b_{1}$ and $a_{2}=$ $-b_{2}$ then the process of thermal emission tends to that limiting geometry where now all exotic energy contributing the Kleinbottle hole with $\left|a_{i}\right|>\left|b_{i}\right|$ is exhausted, leaving a double geometry which is now equivalent to that of a pair of black holes, instead of wormholes, in that the surface embedding the Klein-bottle hole space-time metric (1) always flares inward, being therefore made out of ordinary matter with positive internal energy, which is characterized by a positive temperature; and (C) if any of the two $\varphi_{1}$-intervals making the manifold nonorientable satisfies the condition that $a$ and $b$ become the same and the other reaches a situation where $a=-b$, the final double limiting geometry would be equivalent to that of a wormhole plus a black hole in that the one part of the surface embedding them flares outward and the rest of it flares inward, the former being therefore made of exotic, phantomlike energy with negative temperature, and the latter by ordinary energy with positive temperature.

symmetry the way pointed out before. The existence of such a trapping horizon appeared as the ultimate reason why temperatures and entropies, and actually an entire thermodynamics, are properties pertaining to ringholes or Klein-bottle holes, such as it occurs in wormholes [11].

In what follows, I shall tentatively state the familiar laws that one would expect the Klein-bottle hole thermodynamics should satisfy. Basing on reconciling the laws of 
thermodynamics for ordinary matter and radiation, black holes [19], wormholes [11], a de Sitter universe [20] and warp drives [21] with the existence of a trapping horizon in the Klein-bottle hole, we now seek to tentatively formulate the four laws of Klein-bottle thermodynamics as physical properties that we expect the Klein-bottle holes would have. These laws would be analogous to those of the thermodynamics for ordinary matter and radiation, black holes, wormholes, ringholes [12], the Unruh vacuum [22] or a de Sitter universe, and may be stated as follows. (i) The surface gravity for the two relevant angular regions of a stationary Klein-bottle hole would no longer be strictly constant, in the present case meaning that $\kappa_{i} \equiv \kappa_{i}\left(\varphi_{2}\right)$ (with $i=1,2$ ) remains constant along time for every value of the angle $\varphi_{2}$, not that the $\kappa_{i}$ are kept constant irrespective of the angle $\varphi_{2}$. This would express a zeroth law. (ii) A first law would then state that

$$
d M^{i}=\frac{\kappa_{i}}{8 \pi} d A_{R H}^{i},
$$

that is,

$$
\frac{d M^{i}}{d \varphi_{2}}=\frac{1}{4} \kappa_{i} m_{i}, \quad \frac{d M^{i}}{d \varphi_{1}}=\frac{1}{4} \kappa a_{i} b_{i},
$$

where $\kappa_{i}$ is the surface gravity as given by Eqs. (11) and (12), and $M^{i}=M_{I}^{i}+M_{I I}^{i}$. (iii) If one now assumes the weak energy condition to hold, then the inequality

$$
d A_{K H}^{i} \geq 0,
$$

would express the second law in case that the Klein-bottle hole is placed in an empty environment. For the most realistic situation in which the Klein-bottle hole is surrounded by some ordinary material and/or radiation, as well as gravitationally compact objects, such as black holes, wormholes, ringholes, warp drives or the Unruh vacuum, then this second law had to be generalized to also encompass the entropies of all of these materials and objects [22]. Finally, (iv) the statement that it is not possible to have a Klein-bottle hole with $\kappa_{i}=0$ would amount to the description of a third law.

On the other hand, enclosing a Klein-bottle hole in a box, having perfectly reflecting walls, which also contains arbitrary amounts of ordinary radiation and phantom radiation [23], and then letting the whole system to quantummechanically evolve in time, should always produce an entropy increase, in agreement with the generalized second law [24], no matter whether or not quantum gravity is a time-symmetric theory [25]. However, in order to show that this is really the case, one ought to use an even more careful treatment than that employed when dealing with systems whose components have all positive internal energy and positive temperature [26]. In fact, e.g. it is known [27] that systems which are characterized by a negative temperature are able to only show a finite number of modes available, and this may finally result in an entropy reduction when positive energy is added onto the system.

\section{THERMAL RADIATION FROM INTER-UNIVERSE TIME MACHINES: OBSERVABLE OUTBURSTS}

We have now expressions for the surface gravity corresponding to the different space-time tunneling $\kappa_{G}^{j}$, with $j$ referring to $W H$, wormholes, $R H$, ringholes, and $K H$, Klein-bottle holes, that is $[11,12]$

$$
\kappa_{W H}=\frac{K(r)}{2 r^{2}}-2 \pi r\left(\epsilon-p_{r}\right),
$$

with $K(r)$ the lapse function [1], $\epsilon$ the energy density and $p_{r}$ the radial pressure.

$$
\begin{aligned}
\kappa_{R H}= & \frac{b_{0}^{2}}{2 b\left(a^{2}+b^{2}-2 a b \cos \varphi_{2}\right)}-2 \pi\left(a^{2}+b^{2}\right. \\
& \left.-2 a b \cos \varphi_{2}\right)^{1 / 2}\left(\epsilon-p_{r}\right),
\end{aligned}
$$

where the energy density and the radial pressure depend on the angle $\varphi_{2}$, too. Their combination in Eq. (15) reads [1]

$$
\epsilon-p_{r} \simeq-\frac{c^{4} n^{2} b_{0}^{2}}{8 \pi G r^{3} b^{3}}\left(2+\frac{m a}{n b}+\frac{n b}{m a}\right) .
$$

In the interval $2 \pi-\varphi_{2}^{c}>\varphi_{2}>\varphi_{2}^{c}$ the combination $\epsilon-$ $p_{r}$ becomes negative [11] and hence the surface gravity $\kappa$ is definite positive. In case that $-\varphi_{2}^{c}<\varphi_{2}<\varphi_{2}^{c}$ then [1] $\epsilon-p_{r}>0$ and therefore the generalized surface gravity $\kappa$ becomes definite negative, after passing through $\epsilon-p_{r} \simeq$ 0 on the two angular horizons. Expressions for the surface gravity for Klein-bottle holes are those provided by Eqs. (11) and (12). From all of these expressions for surface gravity we can straightforwardly derive the corresponding expressions for temperature by using the usual relation

$$
T=-\frac{\left.\kappa\right|_{\text {Horizon }}}{2 \pi} .
$$

We consider next the effects that mouth acceleration in the corresponding interuniverse time machines may have on the given expressions for surface gravity, and hence for the radiation temperature and frequency. First of all, we shall restrict ourselves to that study, adding later on the consideration of the further modifications that relativistic Doppler effect may induce on both the temperature and frequency. In this way, we shall use the general expression for the surface gravity in all of the solutions we are considering [11]

$$
\kappa=\frac{1}{2} g^{a b} \partial_{a} \partial_{b} r
$$

where the spherical radius of wormholes $r$ becomes, as we pointed out before, the radius of the circumference described from the hole symmetry axis at the given value of 
angle $\varphi_{2}$ in the case of the toroidal solutions. Now, converting any of the static space-time metrics for the above three tunnelings into the line elements for the corresponding time machines would imply that the $t t$-component of the metric tensor is multiplied by a factor which reads [1,2]

$$
Q=\left[1+g \ell F(\ell) \sin \varphi_{2}\right]^{2},
$$

where $\quad-\infty<\ell<+\infty, \quad g=\gamma^{2} d v / d t \quad$ (with $\quad \gamma=$ $\left.\left(1-v^{2}\right)^{-1 / 2}\right)$ is the acceleration of the mouth which is moving, $F(\ell)$ is a form factor that vanishes on the half of the tunnel whose mouth does not move, $\ell \leq 0$, and rises monotonously from 0 to 1 as one goes leftward or rightward from the throat to the moving mouth, and $\varphi_{2}$ becomes $\theta+$ $\pi / 2$ in the case of wormholes. In deriving this factor we have also used the expressions $d v=g d t / \gamma^{2}$ and $d \gamma=$ $v g \gamma d t$. Now, if we want to keep a $t t$-metric tensor component unity (or more generally $g_{t t}=e^{2 \Phi}$, with $\Phi$ a shift function), we can write the metrics in the purely conformal form

$$
d s_{T M}^{2}=Q\left(-d t^{2}+\frac{d \ell^{2}+d \Pi^{2}}{Q}\right)
$$

in which for the sake of simplicity we have made zero the shift function, $d \Pi^{2}$ is the metric on the unit either twosphere (wormholes) or two-torus (ringholes and Klein-bottle holes), and $Q$ can be interpreted as a well-defined nonsingular conformal factor, $0<Q \leq 1$, because the exotic matter entering the tunnels must satisfy the relativistic requirement $v<1$. In this way, if we take for the hole metric $d s^{2}=d s_{T M}^{2} / Q$, by employing the usual expression for temperature $T=-\left.\kappa\right|_{\text {Horizon }} / 2 \pi$, since the radius $r$ appears to be in all the cases independent of the mouth relative motion, we finally obtain

$$
T_{j}^{T M}=\frac{\left(1-v^{2}+\frac{d v}{d t} \ell F(\ell) \sin \varphi_{2}\right)^{2}}{\left(1-v^{2}\right)^{2}} T_{j},
$$

with $j$ denoting wormhole $(W H)$, ringhole $(R H)$ or Klein-bottle hole $(K H)$ and $\varphi_{2}=\theta+\pi / 2$ in the case of wormholes and $T_{K H} \equiv T_{K H}^{i}, i=1,2$. However, Eq. (19) is not yet the final expression for the temperature of the time machines that correspond to the three types of tunneling we have studied. Indeed, it should still be modified by including a corrective factor arising from the relativistic Doppler effect associated with a moving black body. In fact, the Doppler shift manifests on both the radiating frequency and temperature. The frequency will vary according to

$$
\nu^{D}=\nu \frac{1-v \cos \alpha}{\sqrt{1-v^{2}}}
$$

where $\alpha$ is the angle between the velocity vector and the observer-source direction measured in the reference frame of the source, and the temperature that we finally obtain when including the Doppler effect is given by

$$
T_{j}^{T M / D}=\frac{\left(1-v^{2}+\frac{d v}{d t} \ell F(\ell) \sin \varphi_{2}\right)^{2}}{\left(1-v^{2}\right)^{3 / 2}(1+v)} T_{j},
$$

in which $v>0$ indicates a receding source, and $v<0$ indicates an approaching source. Thus, according to Eq. (20), if we want all the single universe space-times entering the multiverse to be independent of each other, then every interuniverse tunneling network will be emitting a flow of radiation at a rather random temperature which would randomly oscillate between that corresponding to a static tunnel when $v=0$ and a value approaching infinite as $v \rightarrow 1$, with a completely unpredictable cadence. Whenever the mouths of the tunnel accelerate to each other at extremely large speeds, very close to the speed of light, though not reaching it, one would expect the emergence from the furthest confines of our universe of a radiative burst made of just phantom energy in the case of wormholes or a mixture of ordinary and phantom energy in the cases where the interconnecting topology is associated with a torus. The observability from our solar system of such energetic short pulses will depend on the geometric parameters defining the tunnels and on the energetic balance implied by the combination $\epsilon-p_{r}$. The odds for getting a proof of the existence of other universes this way look small, although one could expect that these pulses will induce instantaneous changes on the isotropy of the microwave background in the universe, which perhaps might be observable (see next section).

It is worth noticing that even at the moment when the moving mouth reaches a speed closest to that of light, one could expect that the emitted bursts do not completely destroy the tunneling connection but possibly left it with a minimum-energy geometry governed by the kinds of configurations described in Fig. 2.

\section{CONCLUSIONS AND FURTHER COMMENTS}

We have first studied the thermal properties of static nonorientable Klein-bottle holes which are characterized by two distinct temperatures accordingly to how we go along the trajectory of angle $\varphi_{1}$ around a transversal section of the nonorientable torus. Thermal emission of this nonorientable tunnel consists of radiation made of both phantom particles and ordinary matter, just like in the ringhole case.

Apart from the already pointed out lensing-effect signatures that interconnecting wormholes, ringholes and the Klein-bottle could leave in our own universe out from the existence of other universes [8], we also consider in this paper still another potentially observable phenomenon based on this kind of interuniverse connection: the possible emission of bursts of phantom or phantom and ordinary thermal radiation arising from the final boundaries of our universe. Such a phenomenon would be originated by the utterly random accelerating behavior of the mouths of the considered space-time tunnels and would be more 
energetic as the energy density involved at the pathological solution becomes larger.

Somehow similar dependence of a black body radiation temperatures on velocity was already found to occur in another pathological solution: the two-dimensional superluminal warp drive [21], a space-time construct which can also be transformed into a time machine enclosed in a bubble filled with a thermal radiation whose characteristic temperature rises with the apparent velocity of the ship [21].

Finally, we briefly comment on the odds that the randomly emitted bursts of thermal radiation have to become actually observable, so making the idea of the existence of the multiverse falseable. Of course, it appears quite difficult that with the precision and reach of the instruments now available, the connecting tunnelings be big enough as to have the extremely large amount of exotic matter required to produce a burst that can be detected from the Earth with our current instrumentation, with this made even harder when one considers that the burst energy would dissipate through the large quantity of highly energetic features taking place in our universe during its way to Earth. The possibilities of detecting these phenomena would be considerably increased by using indirect procedures, such as investigating the kind of anisotropies or inhomogeneities in the microwave background that the combined pulses of phantom and ordinary radiation are expected to produce, or the effects that these pulses may have on astronomical objects such as black holes [28] and intrauniversal wormholes [29], decreasing or increasing their size, respectively.

\section{ACKNOWLEDGMENTS}

The author thanks Carmen L. Sigüenza for useful comments and the members of the Estación Ecológica de Biocosmología, in Medellín, Spain, for fruitful conversations. This work was supported by MICINN under Research Project No. FIS2008-06332.
[1] M. S. Morris, K. S. Thorne, and U. Yurtsever, Phys. Rev. Lett. 61, 1446 (1988); M. S. Morris and K. S. Thorne, Am. J. Phys. 56, 395 (1988).

[2] P. F. González-Díaz, Phys. Rev. D 54, 6122 (1996).

[3] P. F. González-Díaz and L. J. Garay, Phys. Rev. D 59, 064026 (1999).

[4] W. A. Hiscock and D. A. Konkowski, Phys. Rev. D 26, 1225 (1982); V. P. Frolov and K. S. Thorne, Phys. Rev. D 39, 2125 (1989); K.S. Thorne, Misner Space as a Prototype for Almost Any Pathology Directions in General Relativity, edited by B.L. Hu, M.P. Ryan, Jr., and C. V. Vishveshwara (Cambridge University Press, Cambridge, United Kingdom, 1993).

[5] S. W. Hawking, Phys. Rev. D 46, 603 (1992).

[6] Li-Xin-Li and J. R. Gott, Phys. Rev. Lett. 80, 2980 (1998).

[7] P. F. González-Díaz, Phys. Rev. D 58, 124011 (1998).

[8] P. F. González-Díaz, Lensing Effects in Ringholes and the Multiverse Cosmology, the Quantum Vacuum, and Zeta Functions, edited by S. D. Odintsov, D. Sáez-Gómez, and S. Xambó-Deschamps (Springer-Verlag, Berlin, Germany, 2011); P. F. González-Díaz and A. Alonso-Serrano, Phys. Rev. D 84, 023008 (2011).

[9] S. Robles-Pérez, and P. F. González-Díaz, Phys. Rev. D 81, 083529 (2010).

[10] P. F. González-Díaz, "The Quantum State of the Universe in the Multiverse" (unpublished).

[11] P. Martín-Moruno and P.F. González-Díaz, Phys. Rev. D 80, 024007 (2009); Classical Quantum Gravity 26, 215010 (2009).

[12] P. F. González-Díaz, Phys. Rev. D 82, 044016 (2010).
[13] S. W. Hawking, Commun. Math. Phys. 43, 199 (1975).

[14] M. Abramowitz and I. A. Stegun, Handbook of Mathematical Functions Dover, New York, 1972).

[15] E. M. Purcell, and R. V. Pound, Phys. Rev. 81, 279 (1951); N. F. Ramsey, Phys. Rev. 103, 20 (1956).

[16] L. H. Ford and T. A. Roman, Phys. Rev. D 60, 104018 (1999).

[17] A. A. Shatskiy, Astronomy Reports 51, 81 (2007); Phys. Usp. 52, 811 (2009); Astronomy Reports 51, 81 (2007); Astron. Zh. 84, 99 (2007); A. A. Shatskiy, I. D. Novikov, and N. S. Kardashev, Phys. Usp. 457 (2008).

[18] P. F. González-Díaz, Phys. Rev. D 68, 084016 (2003).

[19] J. M. Bardeen, B. Carter, and S. W. Hawking, Commun. Math. Phys. 31, 161 (1973).

[20] G. W. Gibbons and S. W. Hawking, Phys. Rev. D 15, 2738 (1977).

[21] P. F. González-Díaz, Phys. Lett. B 653, 129 (2007).

[22] W. G. Unruh, Phys. Rev. D 14, 870 (1976).

[23] P. C. W. Davies and S. A. Fulling, Proc. R. Soc. A 354, 59 (1977).

[24] J. D. Bekenstein, Phys. Rev. D 9, 3292 (1974).

[25] J. A. S. Lima and J.S. Alcaniz, Phys. Lett. B 600, 191 (2004).

[26] G. Izquierdo and D. Pavón, Phys. Lett. B 633, 420 (2006).

[27] I. Brevik, S. Nojiri, S. D. Odintsov, and L. Vanzo, Phys. Rev. D 70, 043520 (2004).

[28] E. Babichev, V. Dokuchaev, and Yu. Eroschenko, Phys. Rev. Lett. 93, 021102 (2004).

[29] P.F. González-Díaz, Phys. Rev. Lett. 93, 071301 (2004). 